\title{
Interaction Design of a Semi-automatic Video Face Annotation System
}

\author{
Cailiang Liu, Tao Xiong, Chenguang Zhang, and Zhibing Wang \\ Hulu LLC 10/F, Building B, ChuangXin Plaza, Tsinghua Science Park, \\ Beijing 100084, P.R. China \\ \{cailiang.liu, tao.xiong, chenguang.zhang, zhibing.wang\}@hulu.com
}

\begin{abstract}
In this paper, we propose an intelligent system that allows people to annotate face tracks of video content. To reduce the workload, we adopt two strategies in the system: 1) visually similar face tracks could be grouped together to reduce human reaction time; 2) face models could be learned to automatically recognize celebrity identities, leaving annotator only simple judgement tasks. With more precise face models, the recognized results require much less time for confirmation. Altogether, these strategies significantly reduce the workload of human annotation/confirmation. Experiments on a very large video repository prove the efficiency and effectiveness of the proposed system.
\end{abstract}

Keywords: User Interfaces, Video Content Analysis, Video Advertisement, Face Track Annotation.

\section{Introduction}

Internet is becoming an important distribution channel for multimedia content. More and more people choose to watch video content via internet, not only because of its rich content, but also because of its free and low cost. Many companies also foresee this big opportunity, i.e., by displaying advertisements to the population to stimulate consumption, they can help product companies profit more, shape and promote their public figure. With limited impression views by the populations, advertising companies have to maximize the click through rate per impression to help product companies profit more. That is, display the advertisement to the most potential buyers. Generally, there are two ways to achieve this: 1) understand users preferences from their online behavior history and display advertisements related to user preference; 2) associate advertisements to related multimedia content. The first way can be regarded as mining stable and long-term preferences. In addition, the second way can be regarded as mining short-term or instant preferences. It could be very useful especially for trailer or commenting videos. Users watching such videos commonly because they concern about the recent development of the story or they want to discuss about the story with the online community. They often like goods (hats, watch, clothes, cars etc) related to celebrities in the video. 
The commercial value of the first way has been mined a lot while the value of the second way is largely underdeveloped. Video content analysis technologies can help find out concept or objects in video. But the researches for the problem is still in early stage [1, 2]. Automatic extraction of general objects or concept is still intractable. Besides advertisement, video content analysis could also help user navigate and locate video content in finer level. As shown in Figure 1 ] video content analysis results could also be used to integrate and summarize information from all over the internet.

Fortunately, after near 10 years' development, the extraction of some specific concepts becomes mature and usable, such as face, pedistrian and trademark detection [3 5]. These concepts are relevant to our motivation. Considering that people are the most frequent subjects in TV episodes or movies, in this paper, we focus on the face recognition problem, which is far more difficult and challenging than just face detection [6, 7]. More specifically, we choose to tackle the problem in a semi-automatic way, by designing an interactive and intelligent annotation system.

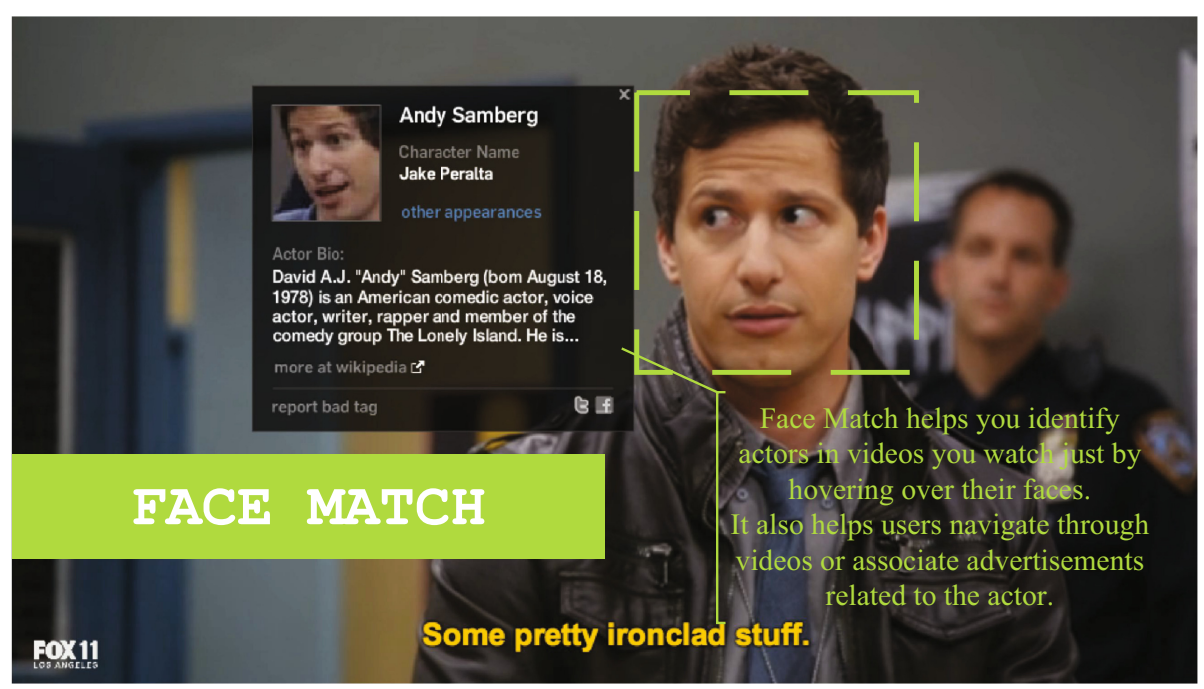

Fig. 1. Face match features on website. Users can see celebrity information by hoving mouse on faces.

There has been user interfaces for face or object annotations $8-12$. However, the existing user interfaces [13, 11] could not tackle our problem: 1) they aim at static faces while we focus on video face track as a basic unit. The face images are more challenging, including occlusion, large variance and severe blur; 2) they lack of a face model for each person, which can automatically annotate faces leveraging existing labels; 3 ) they lack of user interfaces that group visually similar faces to reduce user workload. 
To address the above issues, we propose a semi-automatic video face annotation system. The system is composed of three parts: 1) backend services that extract and process face tracks; 2) annotation user interface; 3) confirmation user interface. For each video, as shown in Figure 2, there are 3 steps for users to label the faces: 1) randomly choose some faces and cluster visually similar faces for user annotation; 2) with these bootstrap information, the system builds face models for each labeled person and automatically tag the rest faces; 3 ) the visually similar tagged faces are clustered together for confirmation. The higher recognition accuracy, the shorter time it will take users to confirm. Summarizing all above, the contributions of the paper are two folded: 1) we propose a semiautomatic way to assist celebrity face annotation for videos; 2) we adopt two strategies to design user interfaces to improve the efficiency of the annotation process.

\subsection{Related Work}

Designing user interfaces for object annotation and pattern recognition has been a long history [14, 10, 8, 15, 16, 12, 13, 11]. In traditional image annotation system, user interfaces are often introduced to label positive and negative examples with respect to some concepts [13, 11]. Because of the difficulties in concept modelling, it requires heavy annotation workload to build an accurate model. Cui et al., Tian et al. [13, 11] propose a system to reduce annotation workload via efficient clustering. Zhang et al. 15] propose a bayesian framework that calculate a name list to reduce annotation time. Later, Zhang et al. [16] propose a framework to calculate the name list for annotating multiple photos at a time. However, it requires people browse the whole album to select the photo set. Suh et al. [17] propose a cluster-based annotation strategy. Different from other work, it also introduces clothes features and event information to improve accuracy.

All the previous work [15, 16, 13, 11] focus on automatic personal album management. However, we are motivated to mine celebrities in TV episodes or movies. In TV episodes or movies, there are diverse face images, e.g., faces with many poses and facial expressions, faces with motion blur or occlusions. This leads to many difficulties in building face models. Fortunately, there are regular celebrities, each celebrity with enough face examples to build accurate models. Moreover, in our scenario, it requires $100 \%$ accuracy to annotate celebrities. So manual confirmation should be involved. Since there are many similar story segments in TV episodes or movies, as shown in Figure 3, introducing clusteringbased annotation could improve the efficiency of the system.

\subsection{Our Method}

With the requirements summerized above, we propose a semi-automatic annotation system to address the problem. The system intelligently combines stateof-art face recognition technologies and efficient user interfaces. For the system overview, please see Figure 2. There are mainly three parts: 
a) Detect and group continuous faces

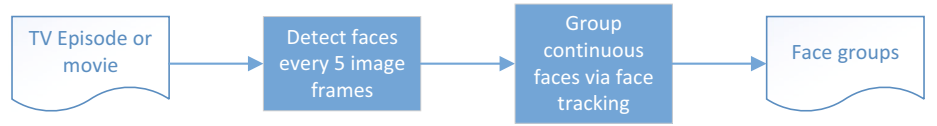

b) Annotation process and user interface design

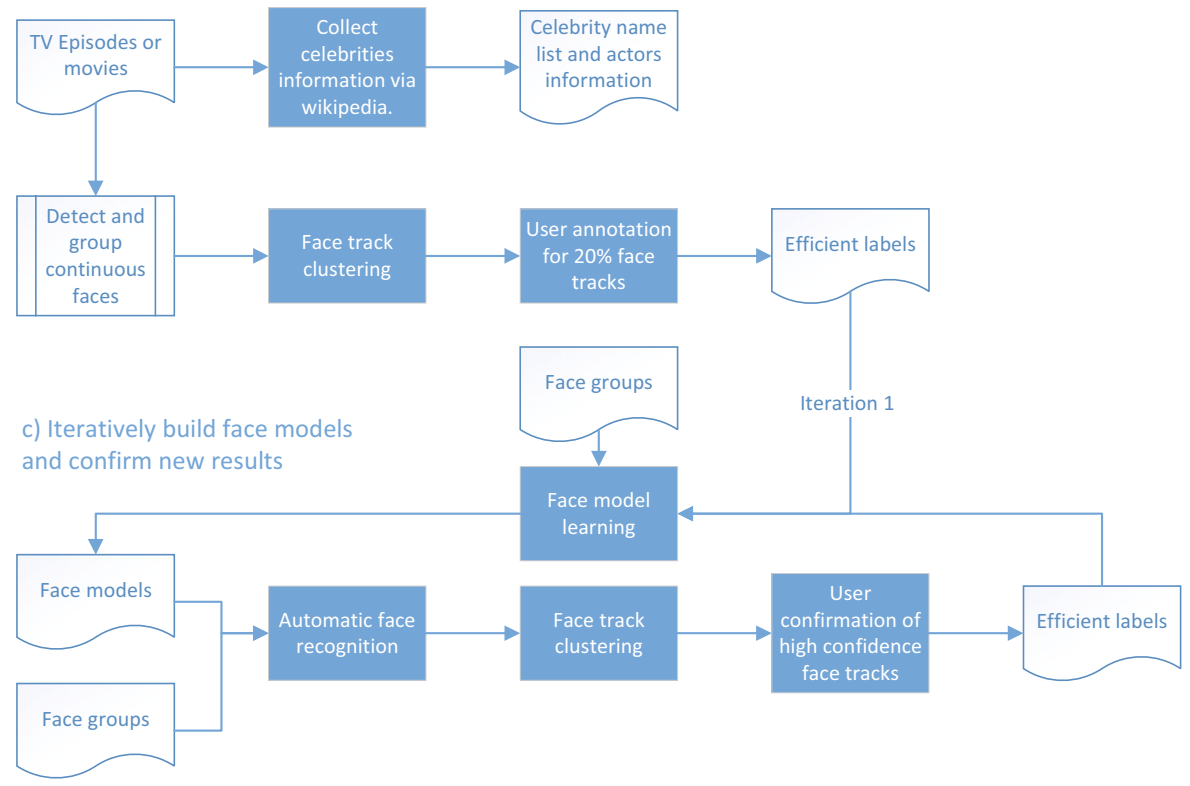

Fig. 2. Overview of the system architect. a) system detects faces and group continuous faces into face groups/tracks for each TV episode/movie; b) system prepares celebrity information for each TV series and select $20 \%$ face track clusters as diverse as possible for initial labels; c) initial user labels are fed to system to learn face models and the automatically recognized results are left for user confirmation.
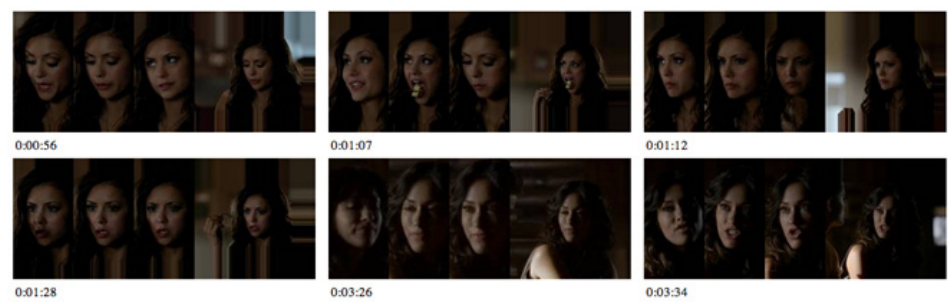

Fig. 3. Similar face tracks of a TV episode. The figure shows track images of the same person from 6 shots. Each cell shows 4 images and the start time of a face track. 
- For each video, the system first detects faces for every 5 frames. Then, the system runs a face tracker to group time continuous faces into face tracks.

- When a series of TV episodes come, the system crawls celebrities information and name list via web. This information will be used in annotation pages. Since the system requires a number of face labels to build accurate models, in the bootsrap stage, $20 \%$ of the face tracks are selected for user annotation. The selected face tracks should be as diverse as posssible to cover as many poses as possible.

- With enough labels, the system builds KNN-based face models [18] for each celebrity. Then the system automatically recognize the rest face tracks. To ensure that the recognition is $100 \%$ accurate, users are required to confirm the recognized results. To improve the efficiency of the confirmation step, the recognized face tracks are also grouped into clusters. Because visually similar faces take much less time for confirmation. In addition, the system allows users to confirm just the face tracks with high confidence. New confirmed labels are fed back to iteratively improve face models.

The paper is composed of five sections. Sectoin 1 introduces background and related work of video face annotation. For the details about how we design the user interfaces and how we combines the state-of-art face recognition technology and user interfaces, please see Section 2 , Section 3 describes the automatic recognition algorithm in detail. Experiments proved the efficiency of our system and user interface design. For the details of experimental results, please see Section 4. Section 5 concludes the efficiency of the system and user interface design.

\section{Interactive Annotation}

We propose user interfaces to assist people annotation and reduce the annotation workload. We design a web portal for annotation tasks. There are three pages in the web portal: a selection page for TV series selection, an annotation page for initial celebrity labels and a confirmation page to confirm automatic recognition results.

The TV series selection page allows people choose which TV serire to annotate. Please see Figure 4 for the detail of the page. The number in the upper-right corner of every TV series indicates the number of videos to annotate. In the web page, users can search TV series via the search box in the navigation bar. Users can also filter TV series by selecting unfinished shows or top shows on the left navigation area. By clilcking the dashboard link, users can view the statistical data.

\subsection{Annotation Page}

Figure 5 shows the page for face tracks annotation. On the left, there is a candidate list of celebrities of the TV series. On the right side, a cluster of face tracks is shown. The rough number of face tracks per cluster is 40 . The first, 


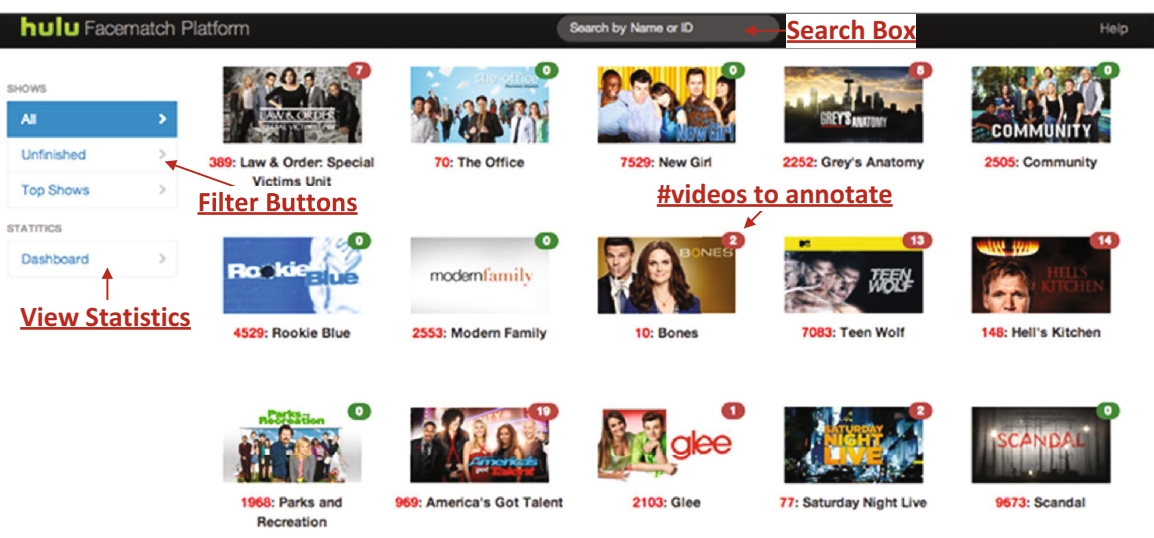

Fig. 4. Series selection page

middle and last faces of a face track are shown to help people verify that it is a complete track. (There may be some errors during motion faces tracking.) To help people identify celebrities, there is also a face image with larger scene context. Possible identities are given in the candidate list. To reduce annotation time, annotators can select multiple face tracks before determining the identity for these face tracks together.

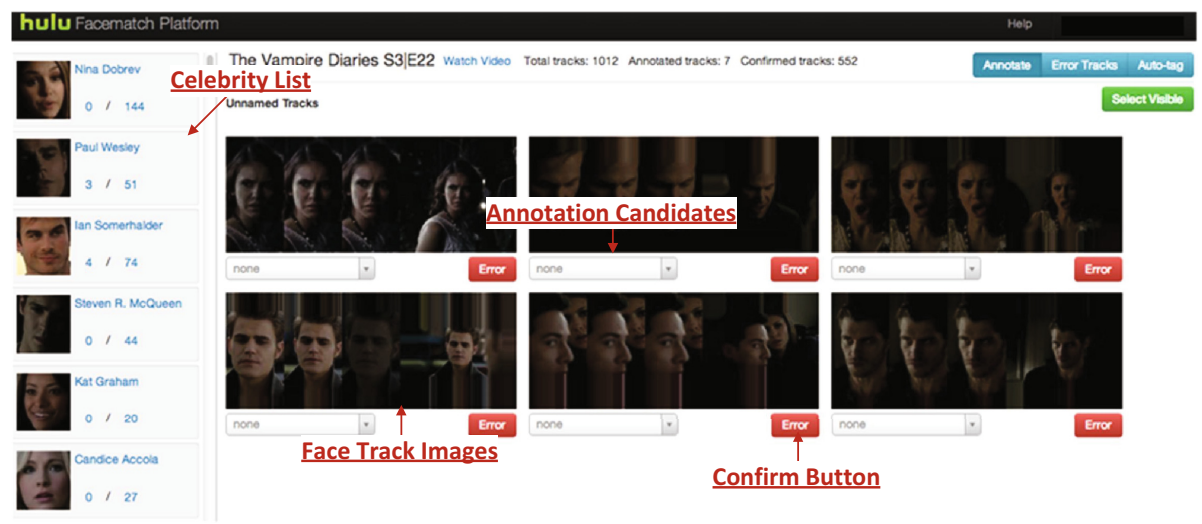

Fig. 5. Clustering-based annotation/confirmation page

\subsection{Confirmation Page}

The confirmation page is used to help people select the correct recognition results, because we require that all the celebrity information shown to user should be $100 \%$ correct. It is similar to the annotation page, as shown in Figure 5 On 
the left panel, there is a celebrity list. For each celebrity, there are a face image, the number of tracks that assigned to him/her and the number of tracks that are confirmed. To reduce workload of user confirmation, users can simply mark the annotation results as 'Error'. Similarly, in the face track area, three face images are shown to represent each face track. In addition, a face image with larger context information is also shown to help identify the celebrity. This helps a lot when the face image is dark.

Because only one click is required for confirmation, the confirmation time is much shorter than annotation time. It is straightforward that it will take less confirmation time when the recognizer is more accurate. For more details of the experiments, please see Section 4. Because there are many similar face tracks and story scenes in a TV episode, grouping these similar face tracks could reduce the reaction time from people. Therefore, we also cluster similar face tracks in confirmation step. Rather than marking each face track right or wrong one by one, it will take less time to select a group of similar face tracks and then confirm it right or wrong.

The recognizer generates a confidence score for each recognition result. Generally, the confidence score is lower for the low quality face tracks or the face tracks that are unknown. The confirmation page automatically rank confident face tracks in the first pages. Users can simply leave the face tracks with low confidence score and continue to the next iteration of automatic recognition and confirmation. With new confirmed face images, the face models become more accurate. The unconfident face tracks could be correctly recognized with better face models. These strategies improves the efficiency of the system.

\section{Algorithms}

In the backend system, as shown in Figure 2 we detect faces for every 5 image frames using a multi-view face detector 19. Then continuous faces are grouped together via a klt face tracking algorithm [20]. A face group or a face track is a basic unit for processing and annotation.

To cluster face tracks, various clustering algorithms could be used, such as kmeans [21], top-down hierarchical clustering [22], agglomerative clustering [23] and so on. We select agglomerative clustering because we can cut at any level to control both the number of face tracks to roughly 40 and the variance of the cluster. Different from single face clustering, each pair of face track are two faces series. Faces series distance should be defined to cluster face tracks. We define the face track distance as the minimal 4-4 faces distance. The median of distances within 16 4-4 face distances is used as final track distance.

To measure face-face distances, the local binary pattern (LBP) face features are used [7]. LBP features measure difference between adjacent pixels. For each pair of pixels, there are only two possible values - higher or lower. Therefore, LBP features are robust to illumination changes. At every pixel location, pixel differences make up an 8-bit code. In a fixed region (eye, nose and so on), we count the number of all kinds of LBP code. This makes up a distribution of codes. 
Besides the robustness to light changes, the distribution also reflects texture properties of facial components.

With new labeled face tracks, the backend system automatically updates face models for every celebrities. Because there are many celebrities, KNN-based models are better choices rather than SVM-based models. It is efficient and requires no training. In recent years, Wright et al. 18] propose a KNN-based classification method using sparse representation. It significantly outperforms other methods. Inspired by the method 18], we also decompose every face as linear combinations of its nearest neighbors. Together with label information, the linear coefficients are used to vote for identity label. The sparsity concentration index (SCI) is used as confidence value for prediction. For every 5-face subset with inner dissimilarities greater than some value, we use the median voting as a recognition candidate. Finally, we take the highest 5 -face voting as the track recognition result. This alleviates the wrong-match problem that a face of person $\mathrm{A}$ is very similar to a face of person $\mathrm{B}$ in feature space.

\section{Experiments}

To evaluate the effectiveness and efficiency of the proposed system, we measure the time used for the annotation tasks. We hire 8 persons to annotate video face tracks. At first, these people are not experts for face annotation. They are not familiar with the celebrities in the TV series. Initially, they are required to annotate only after they finish watching each TV episode. In our video repository, there are totally $186 \mathrm{TV}$ series, 4860 videos. The typical length of a TV episode is about 40 minutes. And each TV episode has about 1294 face tracks averagely.

In the experiments, we measure the annotation time per video (all tracks), annotation time per track, confirmation time per video and confirmation time

\section{Efficiency of Face Match Platform}

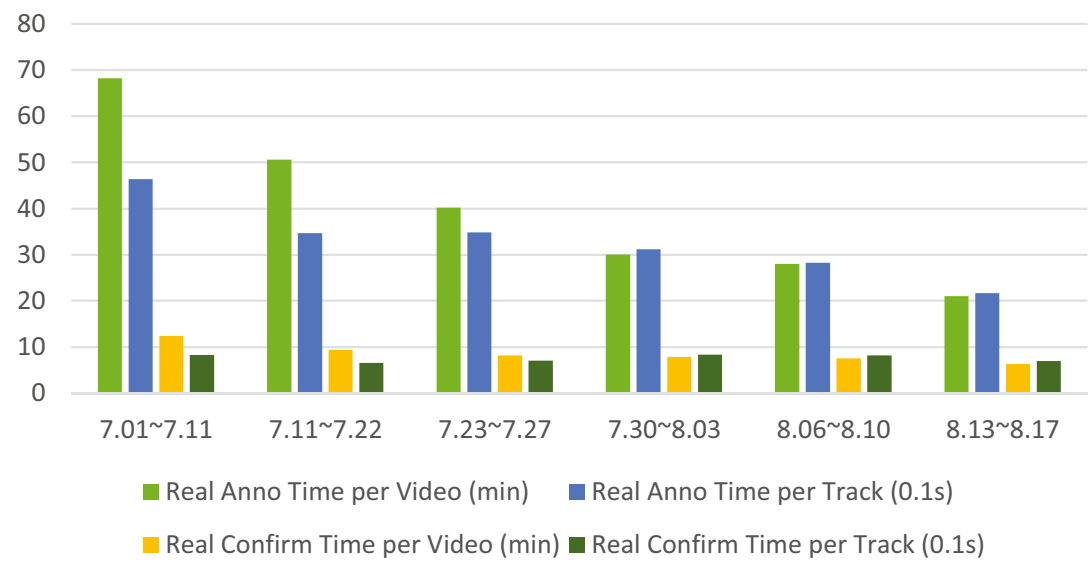

Fig. 6. Efficiency of face match platform 
per track. The system measures user performance at 6 different period, as shown in Figure 6. During these periods, 140 videos are annotated and confirmed per person. As time goes on, users get familiar with celebrities in TV series. With more and more celebrity labels, face models become more precise iteratively. As expected, the average time for annotation tasks per person descreas continuously, as shown in Figure 6. Please also notice that, the confirmation time is significantly smaller than annotation time. It proves the effectiveness of the strategy that we continuously utilize existing celebrity labels and leave the simpler confirmation tasks for users.

\section{Conclusions and Future Work}

Video content analysis is very important for online video websites. Besides advertisement targeting, it also allows internet users to navigate and locate content more easily. In this paper, we propose an intelligent annotation system that allows users to annotate faces for video content. Because celebrities are the most frequent and important subjects in TV episodes. To reduce users workload, we adopt two simple strategies in interaction design: 1) visually similar face tracks could be annotated together to reduce user reaction time; 2) with large amount of existing celebrity labels, precise face models could be learned to help annotate. Experiments proves that the two strategies significantly reduce the user annotation time.

In future, more intelligent algorithms could be proposed to learned preciser models. More face features could be added to backend system to produce more robust and consistent clusters. These are expected to reduce annotation time further. In some TV series, there could be new celebrities unknown to system. Algorithms that automatically discover new celebrities could also be introduced.

\section{References}

1. Rui, Y., Huang, T.S., Ortega, M., Mehrotra, S.: Relevance feedback: a power tool for interactive content-based image retrieval. IEEE Transactions on Circuits and Systems for Video Technology 8(5), 644-655 (1998)

2. Marques, O., Furht, B.: Content Based Image and Video Retrieval, vol. 21. Springer (2002)

3. Viola, P., Jones, M.J.: Robust real-time face detection. International Journal of Computer Vision 57(2), 137-154 (2004)

4. Dalal, N., Triggs, B.: Histograms of oriented gradients for human detection. In: IEEE Computer Society Conference on Computer Vision and Pattern Recognition, CVPR 2005, vol. 1, pp. 886-893. IEEE (2005)

5. Hesson, A., Androutsos, D.: Logo and trademark detection in images using color wavelet co-occurrence histograms. In: IEEE International Conference on Acoustics, Speech and Signal Processing, ICASSP 2008, pp. 1233-1236. IEEE (2008)

6. He, X., Yan, S., Hu, Y., Niyogi, P., Zhang, H.J.: Face recognition using laplacianfaces. IEEE Transactions on Pattern Analysis and Machine Intelligence 27(3), $328-340$ (2005) 
7. Ahonen, T., Hadid, A., Pietikainen, M.: Face description with local binary patterns: Application to face recognition. IEEE Transactions on Pattern Analysis and Machine Intelligence 28(12), 2037-2041 (2006)

8. Nakazato, M., Manola, L., Huang, T.S.: ImageGrouper: Search, annotate and organize images by groups. In: Chang, S.-K., Chen, Z., Lee, S.-Y. (eds.) VISUAL 2002. LNCS, vol. 2314, pp. 129-142. Springer, Heidelberg (2002)

9. Wilhelm, A., Takhteyev, Y., Sarvas, R., Van House, N., Davis, M.: Photo annotation on a camera phone. In: CHI 2004 Extended Abstracts on Human Factors in Computing Systems, pp. 1403-1406. ACM (2004)

10. Grudin, J., Bargeron, D.: Multimedia annotation: an unsuccessful tool becomes a successful framework. Communication and Collaboration Support Systems. TH a. TIEK Okada. Ohmsha (2005)

11. Tian, Y., Liu, W., Xiao, R., Wen, F., Tang, X.: A face annotation framework with partial clustering and interactive labeling. In: IEEE Conference on Computer Vision and Pattern Recognition, CVPR 2007, pp. 1-8. IEEE (2007)

12. Ames, M., Naaman, M.: Why we tag: motivations for annotation in mobile and online media. In: Proceedings of the SIGCHI Conference on Human Factors in Computing Systems, pp. 971-980. ACM (2007)

13. Cui, J., Wen, F., Xiao, R., Tian, Y., Tang, X.: Easyalbum: an interactive photo annotation system based on face clustering and re-ranking. In: Proceedings of the SIGCHI Conference on Human Factors in Computing Systems, pp. 367-376. ACM (2007)

14. Kuchinsky, A., Pering, C., Creech, M.L., Freeze, D., Serra, B., Gwizdka, J.: Fotofile: a consumer multimedia organization and retrieval system. In: Proceedings of the SIGCHI Conference on Human Factors in Computing Systems: The CHI is the Limit, pp. 496-503. ACM (1999)

15. Zhang, L., Chen, L., Li, M., Zhang, H.: Automated annotation of human faces in family albums. In: Proceedings of the Eleventh ACM International Conference on Multimedia, pp. 355-358. ACM (2003)

16. Zhang, L., Hu, Y., Li, M., Ma, W., Zhang, H.: Efficient propagation for face annotation in family albums. In: Proceedings of the 12th Annual ACM International Conference on Multimedia, pp. 716-723. ACM (2004)

17. Suh, B., Bederson, B.B.: Semi-automatic photo annotation strategies using event based clustering and clothing based person recognition. Interacting with Computers 19(4), 524-544 (2007)

18. Wright, J., Yang, A.Y., Ganesh, A., Sastry, S.S., Ma, Y.: Robust face recognition via sparse representation. IEEE Transactions on Pattern Analysis and Machine Intelligence 31(2), 210-227 (2009)

19. Huang, C., Ai, H., Li, Y., Lao, S.: High-performance rotation invariant multiview face detection. IEEE Transactions on Pattern Analysis and Machine Intelligence 29(4), 671-686 (2007)

20. Gejguš, P., Šperka, M.: Face tracking in color video sequences. In: Proceedings of the 19th Spring Conference on Computer Graphics, pp. 245-249. ACM (2003)

21. Hartigan, J.A., Wong, M.A.: Algorithm as 136: A k-means clustering algorithm. Applied Statistics, 100-108 (1979)

22. Johnson, S.C.: Hierarchical clustering schemes. Psychometrika 32(3), 241-254 (1967)

23. Defays, D.: An efficient algorithm for a complete link method. The Computer Journal 20(4), 364-366 (1977) 